\title{
Steady heat conduction analyses using an interpolating element-free Galerkin scaled boundary method
}

\author{
Qinghua Li, Shenshen Chen*, Xinmei Luo \\ School of Civil Engineering and Architecture, East China Jiaotong University,
} Nanchang, 330013, China

\begin{abstract}
Through use of the improved interpolating moving least-squares (IIMLS) shape functions in the circumferential direction of the scaled boundary method based on the Galerkin approach, an interpolating element-free Galerkin scaled boundary method (IEFG-SBM) is developed in this paper for analyzing steady heat conduction problems, which weakens the governing differential equations in the circumferential direction and seeks analytical solutions in the radial direction. The IIMLS method exhibits some advantages over the moving least-squares approximation and the interpolating moving least-squares method because its shape functions possess the delta function property and the involved weight function is nonsingular. In the IEFG-SBM, only a nodal data structure on the boundary is required and the primary unknown quantities are real solutions of nodal variables. Higher accuracy and faster convergence are obtained due to the increased smoothness and continuity of shape functions. Based on the IEFG-SBM, the steady heat conduction problems with thermal singularities and unbounded domains can be ideally modelled. Some numerical examples are presented to validate the availability and accuracy of the present method for steady heat conduction analysis.
\end{abstract}

Keywords: Heat conduction, Scaled boundary method, Element-free Galerkin method, Improved interpolating moving least-squares method, Semi-analytical

\footnotetext{
* Corresponding author.

E-mail address: chenshenshen@tsinghua.org.cn (S. S. Chen).
} 


\section{Introduction}

The analysis of heat conduction problems is very important to engineering and science. However, due to the intrinsic complexity of the corresponding governing equation, analytical solutions for such problems are restricted to simple geometries and boundary conditions. Therefore, more and more efforts have been devoted to analyzing heat conduction problems by utilizing finite element methods (FEM) [1] and boundary element methods (BEM) [2]. Nevertheless, in heat conduction problems with thermal singularity, reasonable accuracy is often impossible or, at least, very costly to obtain by standard numerical methods [3-5]. In order to obtain satisfactory accuracy for heat conduction problems with thermal singularities, special treatments have to be introduced, which may complicate the numerical implementation. Moreover, a difficulty in the application of the FEM for heat conduction problems involving unbounded domain [6] lies in the effective treatment of unbounded domain, because these can not be subdivided into a finite number of elements. For this reason, it is still necessary and urgent to develop novel numerical methods for solving heat conduction problems with thermal singularities and unbounded domains.

The scaled boundary method, developed originally by Wolf and Song [7] in 1996, is an important and attractive computational method as it can represent singularities and unbounded domains accurately and efficiently. It is semi-analytical in the sense of satisfying the equilibrium requirement in the strong form in the radial direction [8]. In this method, a scaled boundary coordinate system is introduced, which scales the boundary to a single point called the scaling center. The governing partial differential equations of various linear problems are weakened in the circumferential direction and then analytical solutions are sought in the radial direction [8]. Thus, only the boundary of the domain is discretized, but no fundamental solution is required. Obviously, 
the computational accuracy of the scaled boundary method may be affected greatly by the discretization approach employed in the circumferential direction [9]. The FEM has been widely used for performing this circumferential discretization and the corresponding method is called the scaled boundary finite element method [10-14]. However, in the scaled boundary finite element method, meshing is still arduous, time consuming and fraught with pitfalls for some problems, especially three-dimensional complicated engineering problems.

As alternative numerical approaches to eliminate the well-known drawbacks in the mesh-based methods, meshless methods have received much attention and gained great success in the field of computational science and engineering. Based on different approximation functions, many kinds of meshless methods have been proposed. Some representative examples are the element-free Galerkin (EFG) method [15, 16], the meshless local Petrov-Galerkin method (MLPG) [17, 18], the reproducing kernel particle method (RKPM) [19] and the point interpolation method (PIM) [20]. Of these meshless methods, the EFG method is a Galerkin discretization technique with the help of shape functions constructed using the moving least-squares (MLS) approximation. To use the MLS approximation, only an array of nodes is required in the domain under consideration. The EFG method exhibits some advantages such as increasing accuracy and rate of convergence, requiring no post-processing to obtain a smooth gradient field $[15,16]$. In order to unite advantages of the scaled boundary method and the EFG method, a novel element-free Galerkin scaled boundary method (EFG-SBM) [21-23] is proposed through the incorporation of the EFG approach into the scaled boundary method. The EFG-SBM is a boundary-type meshless method because no mesh generation is necessary and only a nodal data structure on the boundary is required. It has been demonstrated [21-23] that the EFG-SBM performs better than the conventional scaled boundary 
finite element method. Compared with the MLPG scaled boundary method [24], the EFG-SBM can yield a symmetric stiffness matrix and a more robust solution by retaining the Galerkin approach. However, essential boundary conditions in the EFG-SBM cannot be imposed directly because the obtained shape functions from the MLS approximation lack, in general, the delta function property.

In order to restore the delta function property of the MLS approximation, an interpolating moving least-squares (IMLS) method was developed by Lancaster and Salkauskas [25], which employs specific singular functions as weight functions. In order to obtain higher computational efficiency, a simpler formula of the shape function in the IMLS method was further presented by Ren and Cheng [26-28]. However, the use of IMLS method still has great difficulty in numerical computation because the singularity involved in the weight function complicates the computation of the inverse of the singular matrix and thus increases the computational cost. To overcome this shortcoming, a perturbation technique was introduced by Netuzhylov [29] into the IMLS method. However, a new positive parameter required in this technique always affects the computational accuracy. This led Wang et al. [30, 31] to develop an improved interpolating moving least-squares (IIMLS) method with a nonsingular weight function, which can avoid problems in both the MLS and IMLS schemes. Later, some studies have been conducted to explore the possible application in meshless method [32, 33]. The IIMLS shape functions possess the delta function property and therefore essential boundary conditions can be directly imposed in the IIMLS-based meshless method. In comparison with the IMLS method presented by Lancaster and Salkauskas, the key advantage of the IIMLS method is that it does not require singular weight function and thus any weight function used in the MLS approximation can also be applied in the IIMLS method. In addition, there are less unknown coefficients in the IIMLS method than in the MLS approximation. 
Thus fewer nodes are required in the local influence domain and higher computational accuracy can be reached in the IIMLS-based meshless method.

In this paper, an interpolating element-free Galerkin scaled boundary method (IEFG-SBM) is developed to solve steady heat conduction problems. The shape and test functions in the circumferential direction are constructed by the IIMLS method, which requires only a set of scattered nodes on the boundary. In order not to increase the smoothness of trial functions at corners, the IIMLS interpolation is independently implemented along each edge rather than the entire boundary. The IEFG-SBM is a direct boundary-type meshless method because the basic unknown quantities are the real solutions to the nodal variables and thus essential boundary conditions can be imposed directly. Besides, the scaled boundary equation in temperature is solved analytically using a matrix function solution technique [10]. Numerical examples presented in the end demonstrate that the proposed scheme for solving steady heat conduction problems behaves extremely well, even when the problem involves thermal singularities and unbounded domains.

\section{Governing equations and boundary conditions}

Consider a two-dimensional steady heat conduction problems for a stationary medium on a bounded domain $\Omega$ bounded by $\Gamma$. In the absence of heat generation, the governing differential equation can be written as follows

$$
\nabla^{2} T=0 \quad \text { in } \Omega
$$

with boundary conditions

$$
\begin{array}{cc}
T=\bar{T} & \text { on } S_{1} \\
k T_{, i} n_{i}=\bar{q} & \text { on } S_{2}
\end{array}
$$

where $T$ denotes the temperature; $k$ represents the thermal conductivity; $S_{1}$ and $S_{2}$ are the 
Dirichlet boundary and the Neumann boundary, respectively; $\bar{T}$ and $\bar{q}$ are the prescribed temperature and the prescribed heat flux on the corresponding boundaries, respectively; and $n_{i}$ are the components of the unit outward normal vector to the boundary $S$.

\section{Numerical implementation of the IEFG-SBM}

\subsection{The improved interpolating moving least-squares method}

In the present paper, the element-free Galerkin approach based on the improved interpolating moving least-squares (IIMLS) method [30, 31] is utilized to weaken the governing differential equations in the circumferential direction in the scaled boundary method. The Galerkin approach is utilized here and thus both trial and test functions are constructed by using the same IIMLS method.

The IIMLS scheme in this paper is independently implemented on each piecewise smooth edges, which constitute the entire boundary $S$ naturally. Let us assume that a set of scattered nodes $s_{i}(i=1,2, \cdots, N)$ defined by a random function $T(s)$ are distributed on a single edge, where $s$ is the circumferential coordinate. Let $p_{1}(s), p_{2}(s), \cdots, p_{m}(s)$ denote a given set of basis functions with $p_{1}(s) \equiv 1$. In the present study, the quadratic polynomial basis functions are taken. In order to generate shape functions with the delta function property, we first construct the following basis functions as follows

$$
\hat{p}_{i}(\bar{s})_{s}=p_{i}(\bar{s})-\sum_{I=1}^{n} v\left(s, s_{I}\right) p_{i}\left(s_{I}\right), \quad i=1,2, \cdots, m
$$

where $\bar{s}$ is the point in the local approximation of $s, s_{I}(I=1,2, \cdots, n)$ are the boundary nodes with support domain that cover the point $s$, and

$$
v\left(s, s_{I}\right)=\prod_{J \neq I} \frac{s-s_{J}}{s_{I}-s_{J}}
$$


To obtain the IIMLS scheme for a given function $T(s)$, a new function $\hat{T}(\bar{s})_{s}$ should be first defined as follows

$$
\hat{T}(\bar{s})_{s}=T(\bar{s})-\sum_{I=1}^{n} v\left(s, s_{I}\right) T\left(s_{I}\right)
$$

Based on the moving least-squares (MLS) approximation [25] with the new basis functions, the local approximation $\hat{T}^{h}(\bar{s})_{s}$ of the new function $\hat{T}(\bar{s})_{s}$ can be written as follows

$$
\hat{T}^{h}(\bar{s})_{s}=\sum_{i=1}^{m} \hat{p}_{i}(\bar{s})_{s} \hat{a}_{i}(s)
$$

where $\hat{a}_{i}(s)$ are the unknown coefficients of the new basis. From Eq. (4), we have

$$
\hat{p}_{1}(\bar{s})_{s}=1-\sum_{I=1}^{n} v\left(s, s_{I}\right)=0
$$

Then Eq. (7) becomes

$$
\hat{T}^{h}(\bar{s})_{s}=\sum_{i=2}^{m} \hat{p}_{i}(\bar{s})_{s} \hat{a}_{i}(s)
$$

The $m-1$ coefficients $\hat{a}_{i}(s)(i=2,3, \cdots, m)$ in Eq. (9) can be determined by minimizing a weighted discrete $L^{2}$ norm as follows

$$
\begin{aligned}
J(s) & =\sum_{I=1}^{n} w_{I}(s)\left(\hat{T}^{h}\left(s_{I}\right)_{s}-\hat{T}\left(s_{I}\right)_{s}\right)^{2} \\
& =\sum_{I=1}^{n} w_{I}(s)\left(\sum_{I=1}^{n} v\left(s, s_{I}\right) T\left(s_{I}\right)+\sum_{i=2}^{m} \hat{p}_{i}\left(s_{I}\right)_{s} \hat{a}_{i}(s)-T\left(s_{I}\right)\right)^{2}
\end{aligned}
$$

where $w_{I}(s)$ is a weight function associated with node $s_{I}$. The region in which $w_{I}(s)>0$ is known as the nodal support domain. Since the weight function involved in the IIMLS method may not be singular at any point and thus the commonly used quartic spline weight function is employed here as follows

$$
w_{I}(s)=1-6\left(\frac{\left|s-s_{I}\right|}{r_{I}}\right)^{2}+8\left(\frac{\left|s-s_{I}\right|}{r_{I}}\right)^{3}-3\left(\frac{\left|s-s_{I}\right|}{r_{I}}\right)^{4}, \quad\left|s-s_{I}\right| \leq r_{I}
$$


where $r_{I}$ is the radius of the nodal support domain. Since nodes with uniform spacing $\Delta s$ are introduced in the present work, $r_{I}$ can be computed as follows

$$
r_{I}=\alpha \cdot \Delta s
$$

where $\alpha$, taken as 3.0 here, is the scaling factor controlling the nodal support size.

Solving for $\hat{a}_{i}(s)(i=2,3, \cdots, m)$ by minimizing $J$ in Eq. (10) and substituting it into Eq. (9), the local approximation function can be obtained as

$$
\hat{T}^{h}(\bar{s})_{s}=\hat{\boldsymbol{p}}^{\mathrm{T}}(\bar{s})_{s} \boldsymbol{A}^{-1}(s) \boldsymbol{H}(s) \boldsymbol{T}
$$

where

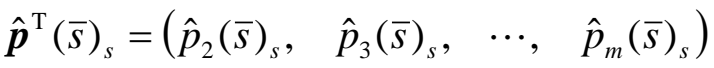

$$
\begin{aligned}
& \boldsymbol{T}=\left(T\left(s_{1}\right), \quad T\left(s_{2}\right), \quad \cdots, \quad T\left(s_{n}\right)\right)^{\mathrm{T}} \\
& \boldsymbol{A}(s)=\sum_{I=1}^{n} w_{I}(s) \hat{\boldsymbol{p}}\left(s_{I}\right)_{s} \hat{\boldsymbol{p}}^{\mathrm{T}}\left(s_{I}\right)_{s} \\
& \boldsymbol{H}(s)=\sum_{I=1}^{n} w_{I}(s) \hat{\boldsymbol{p}}\left(s_{I}\right)_{s}\left(\boldsymbol{e}_{I}-\boldsymbol{v}(s)\right)
\end{aligned}
$$

where $\boldsymbol{e}_{I}$ is a $n$ unit row vector with the $I$ th component being 1 , and

$$
\begin{aligned}
& \hat{\boldsymbol{p}}^{\mathrm{T}}\left(s_{I}\right)_{s}=\left(\begin{array}{llll}
\hat{p}_{2}\left(s_{I}\right)_{s}, & \hat{p}_{3}\left(s_{I}\right)_{s}, & \cdots, & \hat{p}_{m}\left(s_{I}\right)_{s}
\end{array}\right) \\
& \boldsymbol{v}(s)=\left(v\left(s, s_{1}\right), \quad v\left(s, s_{2}\right), \quad \cdots, \quad v\left(s, s_{n}\right)\right)
\end{aligned}
$$

From Eqs. (6) and (13), the global interpolating approximation function of $T(s)$ can be expressed as

$$
T^{h}(s)=\boldsymbol{\Phi}(s) \boldsymbol{T}=\sum_{I=1}^{n} \phi_{I}(s) T\left(s_{I}\right)
$$

where $\boldsymbol{\Phi}(s)$ is a matrix of the shape functions

$$
\begin{aligned}
\boldsymbol{\Phi}(s) & =\left(\phi_{1}(s), \quad \phi_{2}(s), \quad \cdots, \quad \phi_{n}(s)\right) \\
& =\boldsymbol{v}(s)+\boldsymbol{g}^{\mathrm{T}}(s) \boldsymbol{A}^{-1}(s) \boldsymbol{H}(s)
\end{aligned}
$$


and

$$
\begin{aligned}
& \boldsymbol{g}^{\mathrm{T}}(s)=\left(g_{2}(s), \quad g_{3}(s), \cdots, \quad g_{m}(s)\right) \\
& g_{i}(s)=p_{i}(s)-\sum_{I=1}^{n} v\left(s, s_{I}\right) p_{i}\left(s_{I}\right)
\end{aligned}
$$

The partial derivatives of $\boldsymbol{\Phi ( s )}$ can be obtained as follows

$$
\begin{aligned}
\boldsymbol{\Phi}_{, i}(s)= & \boldsymbol{v}_{, i}(s)+\boldsymbol{g}_{, i}^{\mathrm{T}}(s) \boldsymbol{A}^{-1}(s) \boldsymbol{H}(s)+ \\
& \boldsymbol{g}^{\mathrm{T}}(s) \boldsymbol{A}_{, i}^{-1}(s) \boldsymbol{H}(s)+\boldsymbol{g}^{\mathrm{T}}(s) \boldsymbol{A}^{-1}(s) \boldsymbol{H}_{, i}(s)
\end{aligned}
$$

where

$$
\boldsymbol{A}_{, k}^{-1}=-\boldsymbol{A}^{-1} \boldsymbol{A}_{, k} \boldsymbol{A}^{-1}
$$

and the index following a comma is a spatial derivative. Obviously, the shape functions defined in Eq. (21) satisfy the delta function property, namely

$$
\phi_{I}\left(s_{J}\right)=\delta_{I J}
$$

\subsection{IEFG-SBM equation}

In the scaled boundary method, a scaled boundary coordinate system is defined with its origin at the scaling center with the coordinates $\left(\hat{x}_{0}, \hat{y}_{0}\right)$ in a Cartesian coordinate system, as shown in Fig. 1. The circumferential coordinate $s$ specifies a distance anticlockwise around the boundary from an origin on the boundary. The radial coordinate $\xi$ is defined to be zero at the scaling center, unity value at the boundary, and infinity for far field. The conversion between the scaled boundary and Cartesian coordinate system can be written as follows[10-14]

$$
\hat{x}=\hat{x}_{0}+\xi x(s), \quad \hat{y}=\hat{y}_{0}+\xi y(s)
$$

where $(x(s), y(s))$ represents an arbitrary point on the boundary and $(\hat{x}, \hat{y})$ denotes an interior point of the domain. With this geometric mapping, the spatial derivatives in two coordinate systems are related as 


$$
\nabla=\left(\frac{\partial}{\partial x}, \frac{\partial}{\partial y}\right)^{\mathrm{T}}=\boldsymbol{b}_{1}(s) \frac{\partial}{\partial \xi}+\frac{1}{\xi} \boldsymbol{b}_{2}(s) \frac{\partial}{\partial s}
$$

where $\boldsymbol{b}_{1}(s)$ and $\boldsymbol{b}_{2}(s)$ can be written in the form

$$
\boldsymbol{b}_{1}(s)=\frac{1}{|J|}\left\{\begin{array}{c}
y(s)_{, s} \\
-x(s)_{, s}
\end{array}\right\}, \quad \boldsymbol{b}_{2}(s)=\frac{1}{|J|}\left\{\begin{array}{c}
-y(s) \\
x(s)
\end{array}\right\}
$$

with the Jacobian at the boundary being defined as

$$
|J|=x(s) y(s)_{, s}-y(s) x(s)_{, s}
$$

From the IIMLS method, the solution for the temperature at any point can be approximated in the present work by

$$
T(\xi, s)=\boldsymbol{\Phi}(s) \boldsymbol{T}(\xi)
$$

where $\boldsymbol{\Phi}(s)$ denotes the IIMLS shape functions defined in Eq. (21) and the unknown vector $\boldsymbol{T}(\xi)$ represents the nodal temperature function, which is analytical in $\xi$. Combining Eq. (28) and Eq. (31), the derivatives of temperature can be written in terms of the scaled boundary coordinate system as

$$
\nabla T=\left(\frac{\partial T}{\partial x}, \frac{\partial T}{\partial y}\right)^{T}=\boldsymbol{B}_{1}(s) \boldsymbol{T}(\xi)_{, \xi}+\frac{1}{\xi} \boldsymbol{B}_{2}(s) \boldsymbol{T}(\xi)
$$

where the matrices $\boldsymbol{B}_{1}(s)$ and $\boldsymbol{B}_{2}(s)$ are defined as

$$
\boldsymbol{B}_{1}(s)=\boldsymbol{b}_{1}(s) \Phi(s), \quad \boldsymbol{B}_{2}(s)=\boldsymbol{b}_{2}(s) \Phi(s)_{, s}
$$

The Galerkin weak form of the governing Eq. (1) with boundary condition Eqs. (2)-(3) for two-dimensional steady heat conduction problems can be expressed as follows

$$
\int_{\Omega} \delta(\nabla T)^{\mathrm{T}} \cdot k \nabla T \mathrm{~d} \Omega-\int_{S_{2}} \delta T \bar{q} \mathrm{~d} \Gamma=0
$$

Substituting Eqs. (31) and (32) into Eq. (34) yields

$$
\int_{\Omega}\left(\boldsymbol{B}_{1}(s) \delta \boldsymbol{T}(\xi)_{, \xi}+\frac{1}{\xi} \boldsymbol{B}_{2}(s) \delta \boldsymbol{T}(\xi)\right)^{\mathrm{T}} k\left(\boldsymbol{B}_{1}(s) \boldsymbol{T}(\xi)_{, \xi}+\frac{1}{\xi} \boldsymbol{B}_{2}(s) \boldsymbol{T}(\xi)\right) \mathrm{d} \Omega-\delta \boldsymbol{T}_{b}^{\mathrm{T}} \boldsymbol{P}=0
$$


where $\boldsymbol{T}_{b}$ is the nodal temperature vector on the boundary and

$$
\boldsymbol{P}=\int_{S_{2}} \boldsymbol{\Phi}^{\mathrm{T}}(s) \bar{q} \mathrm{~d} s
$$

Expanding the left-hand side of Eq. (35), noting that $\mathrm{d} \Omega=\xi|J| \mathrm{d} \xi \mathrm{d} s$, integrating the domain integrals containing $\delta \boldsymbol{T}(\xi)_{, \xi}$ with respect to $\xi$ using Green's theorem and considering the arbitrariness of the virtual temperature $\delta \boldsymbol{T}(\xi)$, the following relations are yielded

$$
\begin{gathered}
\boldsymbol{P}=\left(\boldsymbol{E}_{0} \boldsymbol{T}(\xi)_{, \xi}+\boldsymbol{E}_{1}^{\mathrm{T}} \boldsymbol{T}(\xi)\right)_{\xi=1} \\
\boldsymbol{P}=\left(-\boldsymbol{E}_{0} \boldsymbol{T}(\xi)_{, \xi}-\boldsymbol{E}_{1}^{\mathrm{T}} \boldsymbol{T}(\xi)\right)_{\xi=1} \\
\boldsymbol{E}_{0} \xi^{2} \boldsymbol{T}(\xi)_{, \xi \xi}+\left(\boldsymbol{E}_{0}+\boldsymbol{E}_{1}^{\mathrm{T}}-\boldsymbol{E}_{1}\right) \xi \boldsymbol{T}(\xi)_{, \xi}-\boldsymbol{E}_{2} \boldsymbol{T}(\xi)=0
\end{gathered}
$$

where $\boldsymbol{E}_{0}, \boldsymbol{E}_{1}$ and $\boldsymbol{E}_{2}$ are the coefficient matrices, which are independent of the radial coordinate $\xi$. They are derived in the following forms

$$
\begin{aligned}
\boldsymbol{E}_{0} & =\int_{S} \boldsymbol{B}_{1}(s)^{\mathrm{T}} k \boldsymbol{B}_{1}(s)|J| \mathrm{d} s \\
\boldsymbol{E}_{1} & =\int_{S} \boldsymbol{B}_{2}(s)^{\mathrm{T}} k \boldsymbol{B}_{1}(s)|J| \mathrm{d} s \\
\boldsymbol{E}_{2} & =\int_{S} \boldsymbol{B}_{2}(s)^{\mathrm{T}} k \boldsymbol{B}_{2}(s)|J| \mathrm{d} s
\end{aligned}
$$

Eqs. (37) and (38) enforce the boundary conditions for bounded domain with $0 \leq \xi \leq 1$ and unbounded domain with $1 \leq \xi \leq \infty$, respectively. Eq. (39) is the generalised scaled boundary equation in temperature, which is a second-order homogeneous differential equation system with respect to the radial coordinate $\xi$.

\subsection{Solution procedure}

At the expense of doubling the number of unknowns, Eq. (39) can be converted to a system of first-order ordinary differential equations as follows[10-14]

$$
\xi \boldsymbol{X}(\xi)_{, \xi}=-\mathbf{Z X}(\xi)
$$

where the variable $\boldsymbol{X}(\xi)$ and the Hamiltonian matrix $\boldsymbol{Z}$ are 


$$
\begin{aligned}
& \boldsymbol{X}(\xi)=\left\{\begin{array}{c}
\boldsymbol{T}(\xi) \\
\boldsymbol{Q}(\xi)
\end{array}\right\}, \\
& \boldsymbol{Z}=\left[\begin{array}{cc}
\boldsymbol{E}_{0}^{-1} \boldsymbol{E}_{1}^{\mathrm{T}} & -\boldsymbol{E}_{0}^{-1} \\
-\boldsymbol{E}_{2}+\boldsymbol{E}_{1} \boldsymbol{E}_{0}^{-1} \boldsymbol{E}_{1}^{\mathrm{T}} & -\boldsymbol{E}_{1} \boldsymbol{E}_{0}^{-1}
\end{array}\right]
\end{aligned}
$$

with $\boldsymbol{Q}(\xi)$ being defined by

$$
\boldsymbol{Q}(\xi)=\boldsymbol{E}_{0} \xi \boldsymbol{T}(\xi)_{, \xi}+\boldsymbol{E}_{1}^{\mathrm{T}} \boldsymbol{T}(\xi)
$$

Block diagonal Schur decomposition [10] is applied to decompose the Hamiltonian matrix $\mathbf{Z}$

such that

$$
\boldsymbol{\psi}^{-1} \mathbf{Z} \boldsymbol{\psi}=\boldsymbol{S}=\operatorname{diag}\left(\boldsymbol{S}_{n}, \boldsymbol{S}_{p}\right)
$$

with the transformation matrix $\boldsymbol{\psi}$ being defined by

$$
\boldsymbol{\psi}=\left[\begin{array}{ll}
\boldsymbol{\psi}_{T n} & \boldsymbol{\psi}_{T p} \\
\boldsymbol{\psi}_{Q n} & \boldsymbol{\psi}_{Q p}
\end{array}\right]
$$

where the matrix $S$ is a block-diagonal matrix in real Schur form and is sorted in ascending order of the real parts of its eigenvalues. The real parts of the eigenvalues of the matrice $\boldsymbol{S}_{n}$ are non-positive and those of the matrice $\boldsymbol{S}_{p}$ are non-negative. $\boldsymbol{S}$ and $\boldsymbol{\Psi}$ can be partitioned into $2 N$ block matrices, respectively. The general solutions for $\boldsymbol{T}(\xi)$ and $\boldsymbol{Q}(\xi)$ follow from Eq. (44) as

$$
\begin{gathered}
\boldsymbol{T}(\xi)=\boldsymbol{\psi}_{T n} \xi^{-\boldsymbol{S}_{n}} \boldsymbol{c}_{n}+\boldsymbol{\psi}_{T p} \xi^{-\boldsymbol{S}_{p}} \boldsymbol{c}_{p} \\
\boldsymbol{Q}(\xi)=\boldsymbol{\psi}_{Q n} \xi^{-\boldsymbol{S}_{n}} \boldsymbol{c}_{n}+\boldsymbol{\psi}_{Q p} \xi^{-\boldsymbol{S}_{p}} \boldsymbol{c}_{p}
\end{gathered}
$$

In a bounded domain $(0 \leq \xi \leq 1)$, the temperatures at $\xi=0$ must remain finite and therefore Eqs. (49a) and (49b) are reduced to

$$
\begin{gathered}
\boldsymbol{T}(\xi)=\boldsymbol{\psi}_{T n} \xi^{-\boldsymbol{S}_{n}} \boldsymbol{c}_{n} \\
\boldsymbol{Q}(\xi)=\boldsymbol{\psi}_{Q n} \xi^{-\boldsymbol{S}_{n}} \boldsymbol{C}_{n}
\end{gathered}
$$

The temperatures on the boundary $\boldsymbol{T}(\xi=1)$ determine the integration constants $\boldsymbol{c}_{n}$ as 


$$
\boldsymbol{c}_{n}=\left(\boldsymbol{\psi}_{T n}\right)^{-1} \boldsymbol{T}(\xi=1)
$$

The stiffness matrix $\boldsymbol{K}_{b}$ for a bounded medium can be determined by substituting Eq. (51) into Eq. (50b) and using Eq. (37) as

$$
\boldsymbol{K}_{b}=\psi_{Q n}\left(\boldsymbol{\psi}_{T n}\right)^{-1}
$$

An unbounded domain is described by $1 \leq \xi \leq \infty$. As the temperatures at $\xi=\infty$ must remain finite, Eqs. (49a) and (49b) become

$$
\begin{gathered}
\boldsymbol{T}(\xi)=\boldsymbol{\psi}_{T p} \xi^{-\boldsymbol{S}_{p}} \boldsymbol{c}_{p} \\
\boldsymbol{Q}(\xi)=\boldsymbol{\psi}_{Q p} \xi^{-\boldsymbol{S}_{p}} \boldsymbol{c}_{p}
\end{gathered}
$$

The integration constants $\boldsymbol{c}_{p}$ can be determined by

$$
\boldsymbol{c}_{p}=\left(\boldsymbol{\psi}_{T p}\right)^{-1} \boldsymbol{T}(\xi=1)
$$

The stiffness matrix $\boldsymbol{K}_{u}$ for an unbounded medium can be determined from Eqs. (38), (53b) and (54) as

$$
\boldsymbol{K}_{u}=-\boldsymbol{\psi}_{Q p}\left(\boldsymbol{\psi}_{T p}\right)^{-1}
$$

\section{Numerical examples}

The present IEFG-SBM has been coded in MATLAB. The validity and strength of the proposed method for solving steady heat conduction problems are demonstrated through different numerical applications including problems with thermal singularities and unbounded domains. The results of these examples are compared with the analytical solutions.

\subsection{A rectangular plate}

The first example refers to the heat conduction problem in a rectangular plate of size $5 \times 10$, as shown in Fig. 2. Its governing equation is written as

$$
\nabla^{2} T=\frac{\partial^{2} T}{\partial x^{2}}+\frac{\partial^{2} T}{\partial y^{2}}=0
$$


The boundary conditions are

$$
\begin{gathered}
T(x, 0)=T(0, y)=0 \\
T(x, 10)=100 \sin (\pi x / 10) \\
\frac{\partial T(5, y)}{\partial x}=0
\end{gathered}
$$

The analytical solution of this problem is

$$
T(x, y)=\frac{100 \sin (\pi x / 10) \sinh (\pi y / 10)}{\sinh (\pi)}
$$

As shown in Fig. 3, uniform nodes are used to discretize the boundary of the problem and $\Delta s$ is the nodal spacing. The center of the plate is chosen as the scaling center. The thermal conductivity is unit value. Table 1 and Table 2 show the numerical solutions for temperature along the vertical line at $x=2.5$ and the horizontal line at $y=5.0$, respectively. In the computation, different numbers of nodes are used. As expected, the numerical results are in excellent agreement with the analytical solutions. In addition, the numerical results also demonstrate that computational accuracy converges with the increase of the number of nodes.

\subsection{Motz problem}

For benchmarking purposes, we consider the Motz problem. Its geometry, boundary conditions and the governing equation are shown in Fig. 4. A singularity arises at $x=y=0$, where the boundary condition suddenly changes from $T=0$ to $\frac{\partial T}{\partial n}=0$. The solution in the vicinity of the singular point is given by [34]

$$
T_{\text {exact }}=\sum_{n=0}^{\infty} b_{n} r^{(2 n+1) / 2} \cos ((2 n+1) \theta / 2)
$$

where

$$
\begin{aligned}
& b_{0}=401.162 ; b_{1}=87.656 ; b_{2}=17.238 ; b_{3}=-8.071 ; \\
& b_{4}=1.440 ; b_{5}=0.331 ; b_{6}=0.275 ; \cdots
\end{aligned}
$$


In order to evaluate the computed error in the domain, the following relative error norm is employed here [35]

$$
\eta=\frac{\left\|T_{\text {exact }}-T_{\text {numer }}\right\|_{L_{2}}}{\left\|T_{\text {exact }}\right\| \|_{L_{2}}} \times 100 \%, \quad\|T\|_{L_{2}}=\left(\int_{\Omega} T^{2} \mathrm{~d} \Omega\right)^{\frac{1}{2}}
$$

where $T_{\text {exact }}$ and $T_{\text {numer }}$ represent respectively the exact and the numerical solutions.

The IEFG-SBM model is presented in Fig. 5. The scaling center is chosen at the origin of the coordinate and therefore the boundaries passing through the scaling center are not discretized. As shown in Fig. 5, only 13 boundary nodes are introduced. Fig. 6 shows the numerical and analytical solutions for temperature along the vertical line at $x=0.0$. As can be seen, the results of the IEFG-SBM agree well with the analytical solutions. In addition, the computed relative error norm defined in Eq. (63) is $0.0355 \%$, which further demonstrates that the IEFG-SBM can produce extraordinarily accurate solutions for this problem even with very few nodes, as the singularity is modelled analytically.

\subsection{A semi-infinite problem}

An attractive advantage of the IEFG-SBM is that it is well suited to analyse the problems with unbounded domain. As a result, the heat conduction problem in a semi-infinite domain $y \geq 0$, as shown in Fig. 7, is considered in the third example. In this problem, the edge $y=0$ is kept at a temperature of zero, except for the segment $-1<x<1$, where it is kept at a temperature of unit. The analytical solution of this problem is as follows [23]:

$$
T=\frac{1}{\pi} \arctan \left(\frac{2 y}{x^{2}+y^{2}-1}\right)
$$

The IEFG-SBM model of this problem is shown in Fig. 8, where three subdomains are employed, one unbounded and two bounded. The defining curves of the first bounded domain are 
$\mathrm{O}_{3} \mathrm{E}$, EB, and BA with scaling center $\mathrm{O}_{1}$, the defining curves of the second bounded domain are DC, $\mathrm{CE}$, and $\mathrm{EO}_{3}$ with scaling center $\mathrm{O}_{2}$, and the defining curves of the unbounded domain are DC, $\mathrm{CB}$, and BA with scaling center $\mathrm{O}_{3}$. Note that $\mathrm{O}_{1}$ and $\mathrm{O}_{2}$ are chosen as scaling centers so that singularities arising at these two points can be ideally modelled. A total of 29 uniformly distributed boundary nodes are introduced. In Fig. 9, computational results for the temperature at $y=1$ by the IEFG-SBM are depicted. For comparison, the corresponding analytical solutions are also plotted in the same graph. It is observed from Fig. 9 that the agreement appears to be very good. Table 3 lists the variation of temperature with coordinate $y$ at $x=0$ comparing the IEFG-SBM solutions and the analytical solutions, which demonstrates that the IEFG-SBM can produce extraordinarily accurate solutions from the near field to the far field. Note that the number of nodes required for this complex problem is still rather small.

\section{Conclusion}

In this paper, a simple and effective formulation of the direct boundary-type meshless method, called interpolating element-free Galerkin scaled boundary method (IEFG-SBM), is presented for two-dimensional steady heat conduction analysis. The numerical results indicate that using the IEFG-SBM to carry out steady heat conduction analyses is effective and appealing. In particular, the IEFG-SBM is also well suitable to deal with heat conduction problems involving thermal singularities and unbounded domains. The primary remarks are concluded underneath:

(1) The IEFG-SBM provides a new approach to establish a boundary-type meshless method. In the IEFG-SBM, no mesh generation is necessary and only a nodal data structure on the boundary is required, which constitutes a big contrast to the scaled boundary finite element method. Due to the increased smoothness and continuity of shape functions, smooth gradient fields can be 
obtained without post-processing and the advantages of accuracy, smoothness and convergence of the EFG method are retained.

(2) In comparison with the EFG-SBM, the IIMLS method is used in the IEFG-SBM to form shape functions in the circumferential direction and consequently essential boundary conditions can be directly imposed without any special treatments. Besides, any weight function used in the MLS approximation can be utilized in the IIMLS method and thus the difficulty arising from singularity of the weight function in the IMLS method can be effectively avoided. Compared with the MLS approximation, there exist fewer unknown coefficients in the IIMLS method, which leads to the fact that fewer nodes are required in the local influence domain, and higher computational accuracy is achieved. Therefore, it is reasonable and feasible to employ the IIMLS method to construct shape functions in the circumferential direction of the scaled boundary method.

\section{Acknowledgments}

The support of National Science Foundation of China (21466012 and 11462006), Foundation of Jiangxi Educational Committee (KJLD14041) and Foundation of East China Jiaotong University (09130020) are gratefully acknowledged.

\section{References}

[1] E. C. Romao, L. F. M. Moura, Galerkin and least squares methods to solve a 3D convection-diffusion-reaction equation with variable coefficients, Numer. Heat Transfer A. 61 (2012) 669-698.

[2] B. Vick, D. J. Nelson, The boundary element method applied to freezing and melting problems, Numer. Heat Transfer B 24 (1993) 263-277.

[3] M. Arad, Z. Yosibash, G. Ben-Dor, A. Yakhot, Computing flux intensity factors by a boundary 
method for elliptic equations with singularities, Commun. Numer. Meth. Engng. 14 (1998) 657-670.

[4] N. K. Mukhopadhyaya, S. K. Maiti, A. Kakodkar, Effect of modelling of traction and thermal singularities on accuracy of SIFS computation through modified crack closure integral in BEM, Eng. Fracture Mech. 64 (1999) 141-159.

[5] N. N. V. Prasad, M. H. Aliabadi, D. P. Rooke, Effect of thermal singularities on stress intensity factors: edge crack in rectangular and circular plate, Theor. Appl. Fracture Mech. 24 (1996) 203-215.

[6] M. Demidem, Analysis of Heat Transfer Problems by Coupling of Finite and Infinite Element, Application of Codes, Design and Regulations, Thomas Telford, 2005, pp. 375-383.

[7] J. P. Wolf, C. Song, Finite-Element Modelling of Unbounded Media, John Wiley, Chichester, UK, 1996.

[8] J. P. Wolf, The Scaled Boundary Finite Element Method, John Wiley, Chichester, UK, 2003.

[9] A. J. Deeks, C. E. Augarde, A meshless local Petrov-Galerkin scaled boundary method, Comput. Mech. 36 (2005) 159-170.

[10] C. Song, A matrix function solution for the scaled boundary finite element equation in statics, Comput. Methods Appl. Mech. Eng. 193 (2004) 2325-2356.

[11] Z. C. Sun, E. T. Ooi, C. Song, Finite fracture mechanics analysis using the scaled boundary finite element method, Eng. Fract. Mech. 134 (2015) 330-353.

[12] C. Song, The scaled boundary finite element method in structural dynamics, Int. J. Numer. Methods Eng. 77 (2009) 1139-1171.

[13] Z. J. Yang, A. J. Deeks, A frequency-domain approach for modelling transient elastodynamics 
using scaled boundary finite element method, Comput. Mech. 40 (2007) 725-738.

[14] A. J. Deeks, J. Wolf, A virtual work derivation of the scaled boundary finite element method for elastostatics, Comput. Mech. 28 (2002) 489-504.

[15] T. Belytschko, Y. Y. Lu, L. Gu, Element-free Galerkin methods, Int. J. Numer. Meth. Eng. 37 (1994) 229-256.

[16] Y. J. Deng, C. Liu, M. J. Peng, Y. M. Cheng, The interpolating complex variable element-free Galerkin method for temperature field problems, Int. J. Appl. Mech. 7 (2015) 1550017.

[17] S. N. Atluri, T. Zhu, A new meshless local Petrov-Galerkin (MLPG) approach in computational Mechanics, Comput. Mech. 22 (1998) 117-127.

[18] M. Shibahara, S. N. Atluri, The meshless local Petrov-Galerkin method for the analysis of heat conduction due to a moving heat source, in welding, Int. J. Therm. Sci. 50 (2011) 984-992.

[19] Y. X. Qin, Y. M. Cheng, Reproducing kernel particle boundary element-free method for elasticity, Acta. Phys. Sin. 55 (2006) 3215-3222.

[20] G. R. Liu, Y. T. Gu, a point interpolation method for two dimensional solid, Int. J. Numer. Methods Eng. 50 (2001) 937-951.

[21] Y. Q. He, H. T. Yang, A. J. Deeks, An element-free Galerkin (EFG) scaled boundary method, Finite Elem. Anal. Design 62 (2012) 28-36.

[22] Y. Q. He, H. T. Yang, A. J. Deeks, Determination of coefficients of crack tip asymptotic fields by an element-free Galerkin scaled boundary method, Fatigue Fracture Eng. Mater. Struct. 35 (2012) 767-785.

[23] Y. Q. He, H. T. Yang, A. J. Deeks, An element-free Galerkin scaled boundary method for steady-state heat transfer problems, Numer. Heat Transfer B 64 (2013) 199-217. 
[24] A. J. Deeks, C. E. Augarde, A meshless local Petrov-Galerkin scaled boundary method, Comput. Mech. 36 (2005) 159-170.

[25] P. Lancaster, K. Salkauskas, Surface generated by moving least squares methods, Math. Comput. 37 (1981) 141-158.

[26] H. P. Ren, Y. M. Cheng, W. Zhang, An improved boundary element-free method (IBEFM) for two-dimensional potential problems, Chin. Phys. B 18 (2009) 4065-4073.

[27] H. P. Ren, Y. M. Cheng, The interpolating element-free Galerkin (IEFG) method for two-dimensional elasticity problems, Int. J. Appl. Mech. 3 (2011) 735-758.

[28] H. P. Ren, Y. M. Cheng, The interpolating element-free Galerkin (IEFG) method for two-dimensional potential problems, Eng. Anal. Bound. Elem. 36 (2012) 873-880.

[29] H. Netuzhylov, Enforcement of boundary conditions in meshfree methods using interpolating moving least squares, Eng. Anal. Bound. Elem. 32 (2008) 512-516.

[30] J. F. Wang, F. X. Sun, Y. M. Cheng, An improved interpolating element-free Galerkin method with a nonsingular weight function for two-dimensional potential problems, Chin. Phys. B 21 (2012) 090204.

[31] J. F. Wang, J. F. Wang, F. X. Sun, Y. M. Cheng, An interpolating boundary element-free method with nonsingular weight function for two-dimensional potential problems, Int. J. Comput. Methods 10 (2013) 1350043.

[32] X. L. Li, An interpolating boundary element-free method for three-dimensional potential Problems, Appl. Math. Model 39 (2015) 3116-3134.

[33] X. L. Li, A meshless interpolating Galerkin boundary node method for stokes flows, Eng. Anal. Bound. Elem. 51 (2015) 112-122. 
[34] Z. Yosibash, B. Schiff, Superelements for the finite element solution of two-dimensional elliptic problems with boundary singularities, Finite Elem. Anal. Design. 26 (1997) 315-335.

[35] X. Liu, C. K. Lee, S. C. Fan, On using enriched cover function in the partition-of-unity method for singular boundary-value problems, Comput. Mech. 29 (2002) 212-225.

\section{Captions}

\section{Figures}

Fig. 1. The scaled boundary coordinate system

Fig. 2. Heat conduction in a rectangular plate

Fig. 3. IEFG-SBM model of example 1

Fig. 4. Motz problem

Fig. 5. IEFG-SBM model of example 2

Fig. 6. IEFG-SBM solutions for temperature at $x=0$

Fig. 7. Heat conduction in a semi-infinite domain

Fig. 8. IEFG-SBM model of example 3

Fig. 9. The variation of temperature at $y=1$

\section{Tables}

Table 1. IEFG-SBM solutions for temperature at $x=2.5$

Table 2. IEFG-SBM solutions for temperature at $y=5.0$

Table 3. The variation of temperature at $x=0.0$ 
Figure 1

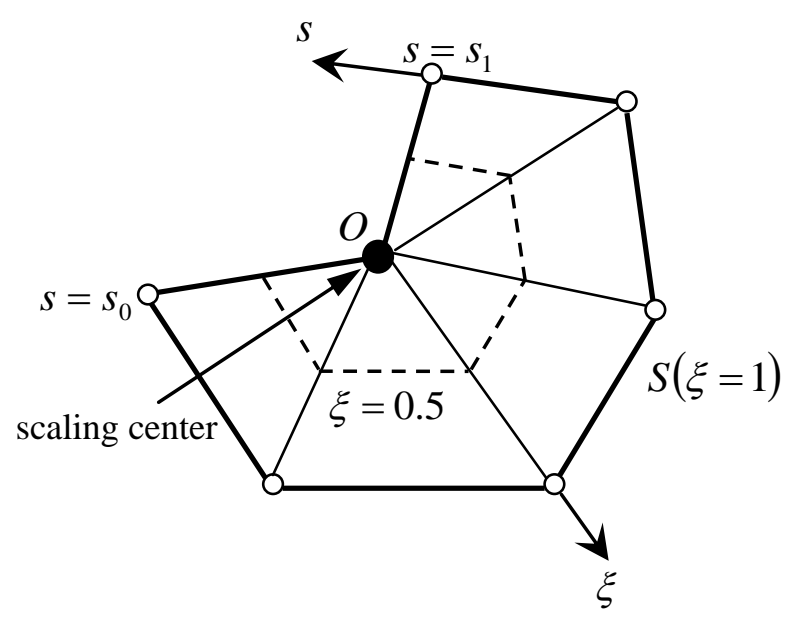

Fig. 1. The scaled boundary coordinate system 
Figure 2

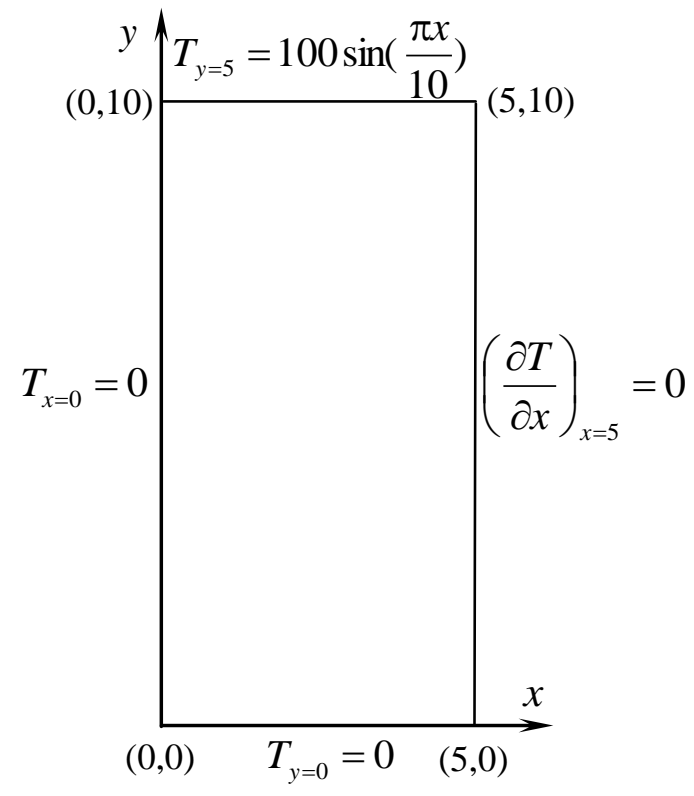

Fig. 2. Heat conduction in a rectangular domain 
Figure 3

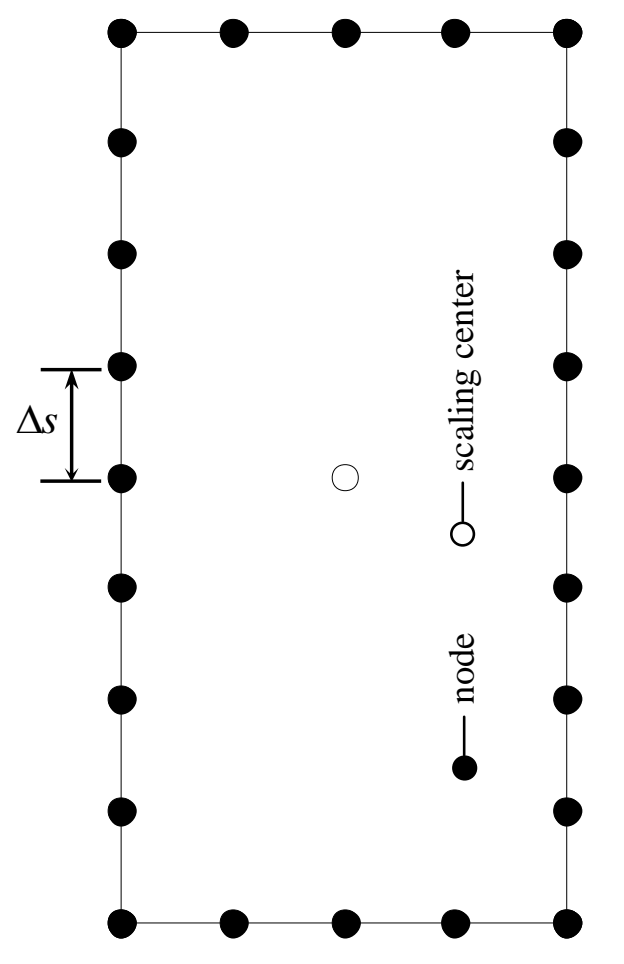

Fig. 3. IEFG-SBM model of example 1

(1)

Fig. 3. IEFG-SBM model of example 1

Figure 3

1

(a)

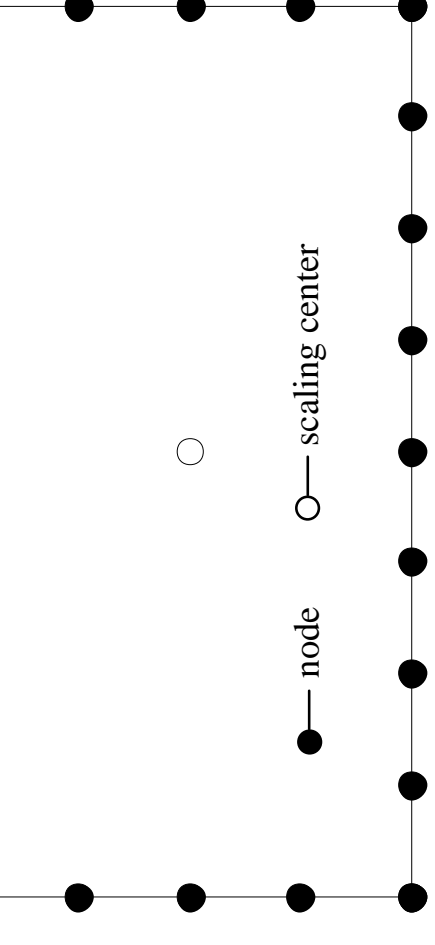

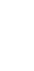

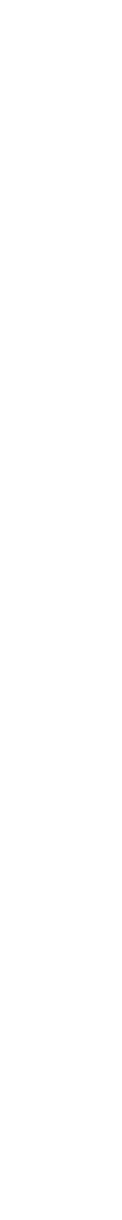


Figure 4

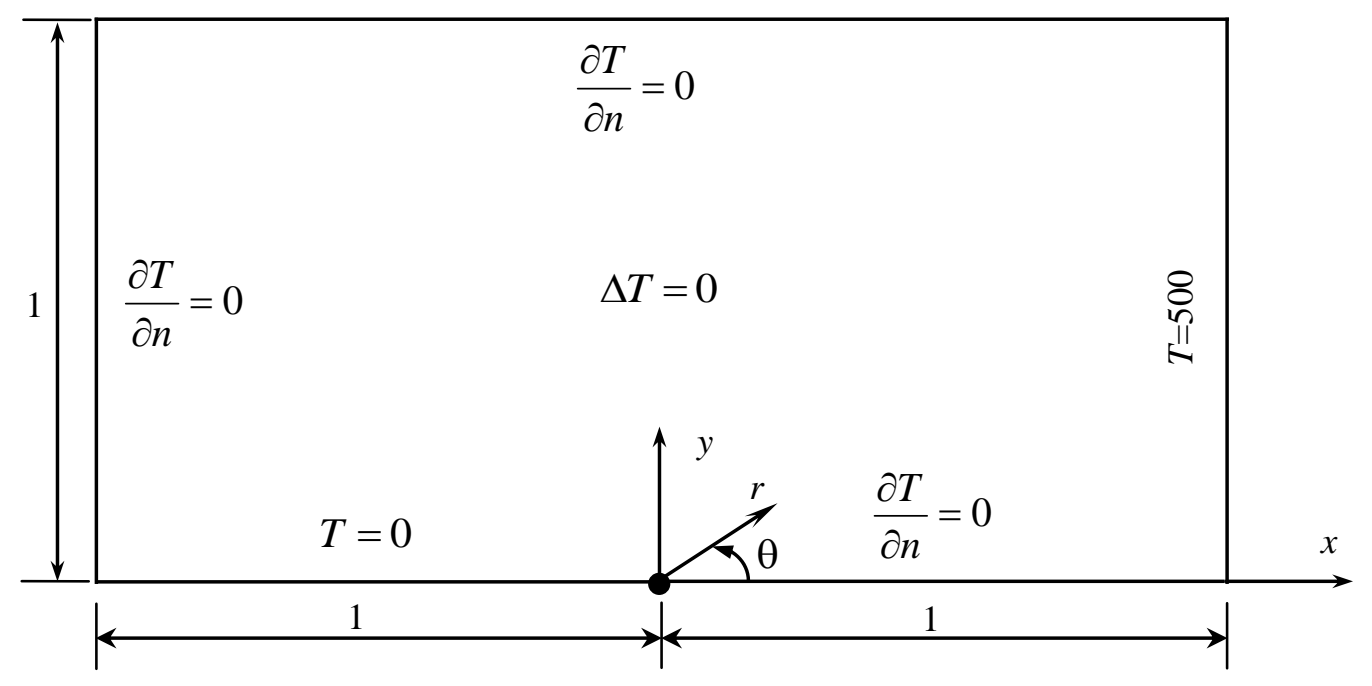

Fig. 4. Motz problem 


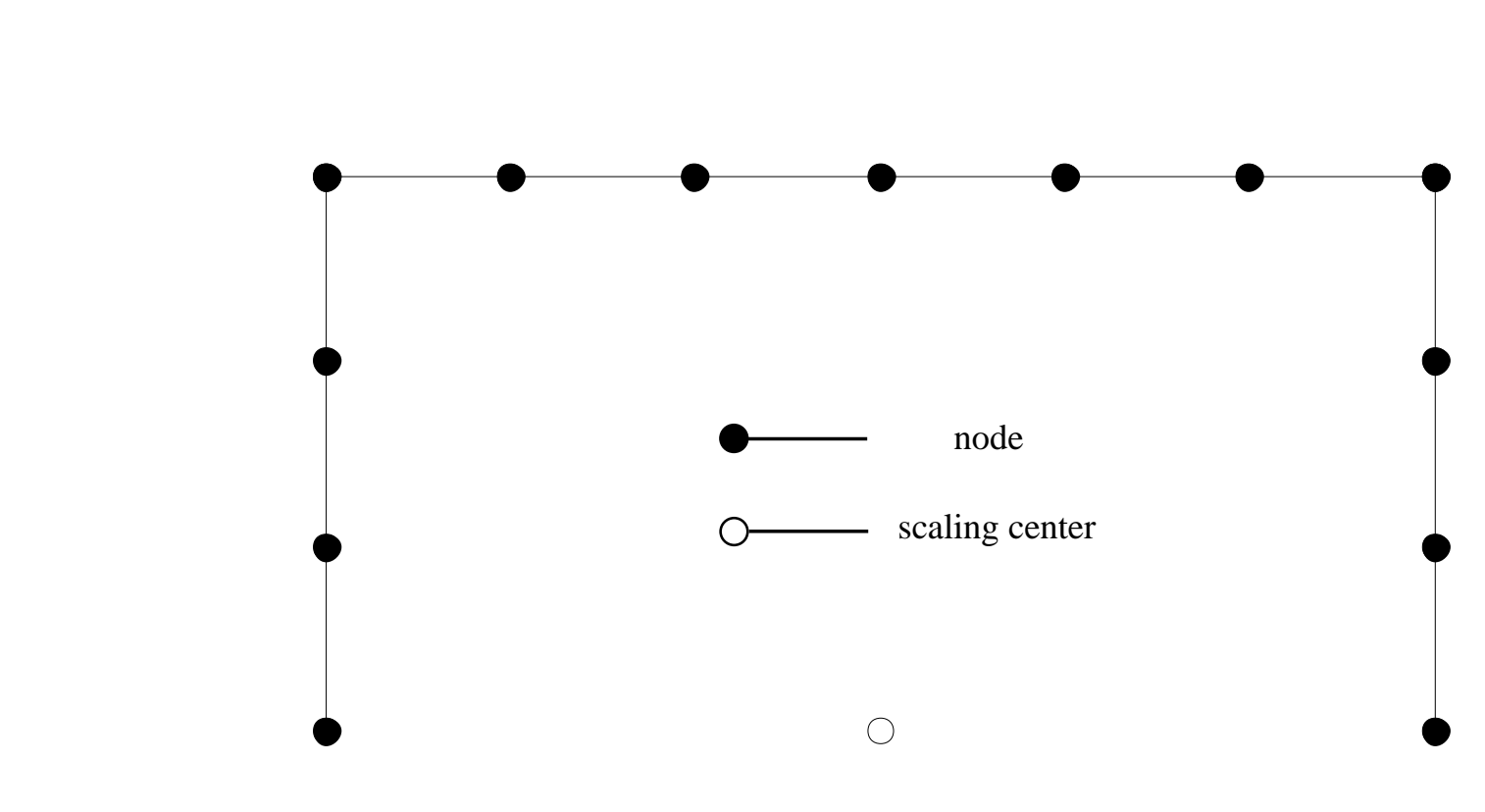

Fig. 5. IEFG-SBM model of example 2

Figure 5

Figure 5

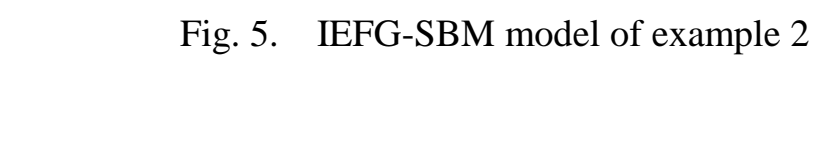

(1)

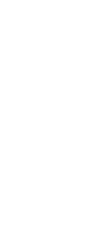


Figure 6

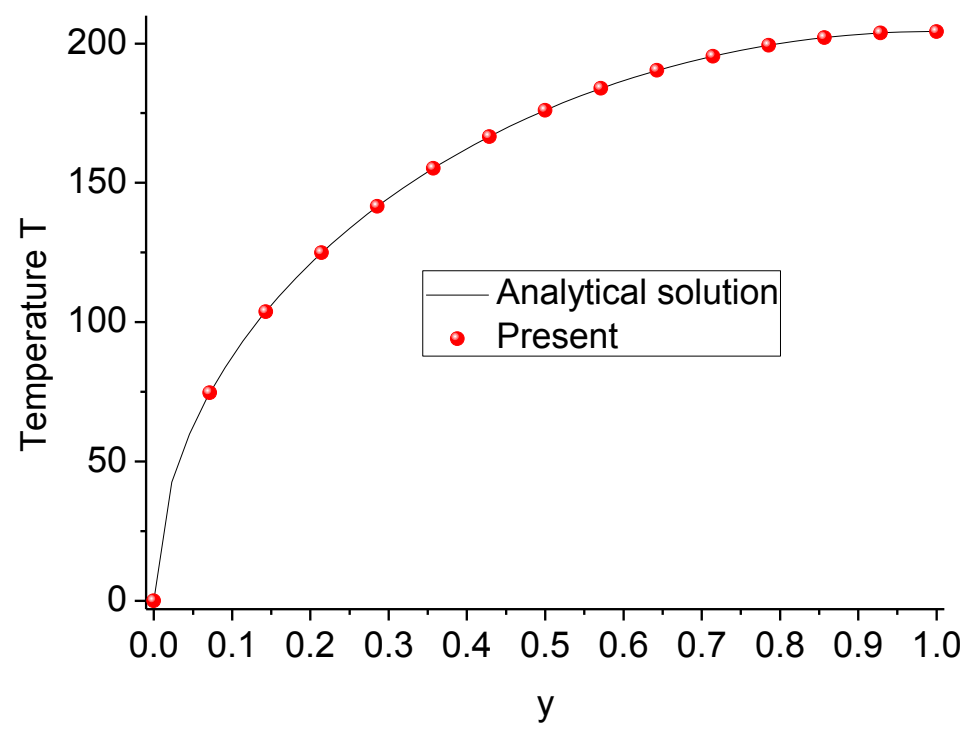

Fig. 6. IEFG-SBM solutions for temperature at $x=0$ 
Figure 7

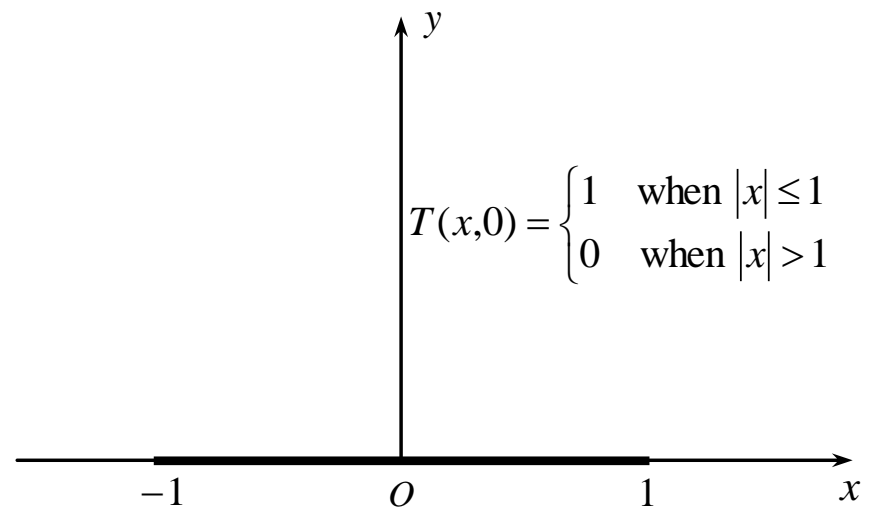

Fig. 7. Heat conduction in a semi-infinite domain 
Figure 8

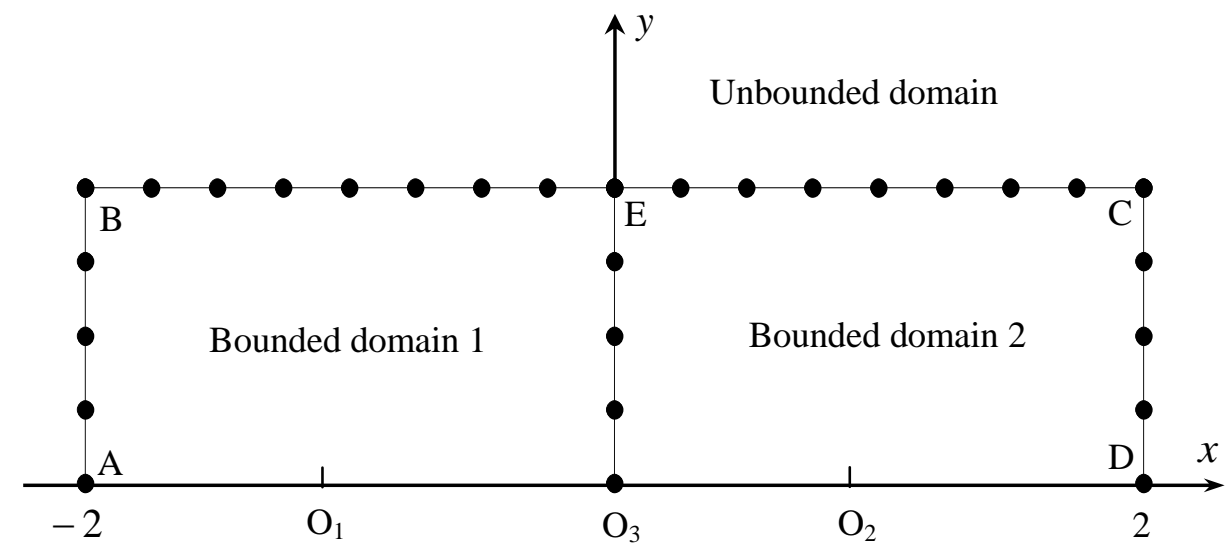

Fig. 8. IEFG-SBM model of example 3 
Figure 9

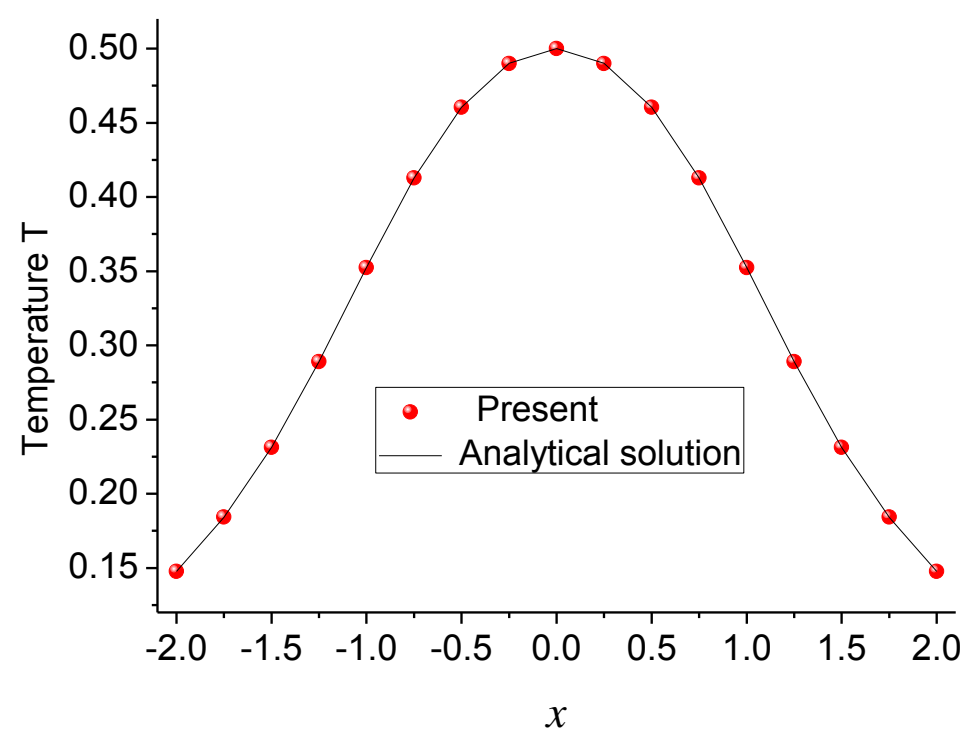

Fig. 9. The variation of temperature at $y=1$ 
Table 1. IEFG-SBM solutions for temperature at $x=2.5$

\begin{tabular}{|c|c|c|c|c|c|c|c|}
\hline \multirow{2}{*}{$y$} & \multicolumn{2}{|c|}{$\begin{array}{c}24 \text { nodes } \\
(\Delta s=1.25)\end{array}$} & \multicolumn{2}{|c|}{$\begin{array}{c}48 \text { nodes } \\
(\Delta s=0.625)\end{array}$} & \multicolumn{2}{|c|}{$\begin{array}{c}96 \text { nodes } \\
(\Delta s=0.3125)\end{array}$} & \multirow{2}{*}{ Analytical } \\
\hline & Present & Error $(\%)$ & Present & Error (\%) & Present & Error (\%) & \\
\hline 0.0 & 0.0000 & 0.0000 & 0.0000 & 0.0000 & 0.0000 & 0.0000 & 0.0000 \\
\hline 1.0 & 1.9561 & 0.0409 & 1.9554 & 0.0051 & 1.9553 & 0.0000 & 1.9553 \\
\hline 2.0 & 4.1065 & 0.0317 & 4.1053 & 0.0024 & 4.1052 & 0.0000 & 4.1052 \\
\hline 3.0 & 6.6656 & 0.0285 & 6.6637 & 0.0000 & 6.6637 & 0.0000 & 6.6637 \\
\hline 4.0 & 9.8880 & 0.0283 & 9.8853 & 0.0010 & 9.8852 & 0.0000 & 9.8852 \\
\hline 5.0 & 14.0945 & 0.0291 & 14.0905 & 0.0007 & 14.0904 & 0.0000 & 14.0904 \\
\hline 6.0 & 19.7033 & 0.0279 & 19.6979 & 0.0005 & 19.6978 & 0.0000 & 19.6978 \\
\hline 7.0 & 27.2722 & 0.0257 & 27.2655 & 0.0011 & 27.2652 & 0.0000 & 27.2652 \\
\hline 8.0 & 37.5554 & 0.0253 & 37.5462 & 0.0008 & 37.5459 & 0.0000 & 37.5459 \\
\hline 9.0 & 51.5762 & 0.0260 & 51.5630 & 0.0004 & 51.5628 & 0.0000 & 51.5628 \\
\hline 10.0 & 70.7107 & 0.0000 & 70.7107 & 0.0000 & 70.7107 & 0.0000 & 70.7107 \\
\hline
\end{tabular}


Table 2. IEFG-SBM solutions for temperature at $y=5.0$

\begin{tabular}{|c|c|c|c|c|c|c|c|}
\hline \multirow{2}{*}{$x$} & \multicolumn{2}{|c|}{$\begin{array}{c}24 \text { nodes } \\
(\Delta s=1.25) \\
\end{array}$} & \multicolumn{2}{|c|}{$\begin{array}{c}48 \text { nodes } \\
(\Delta s=0.625)\end{array}$} & \multicolumn{2}{|c|}{$\begin{array}{c}96 \text { nodes } \\
(\Delta s=0.3125)\end{array}$} & \multirow{2}{*}{ Analytical } \\
\hline & Present & Error (\%) & Present & Error (\%) & Present & Error (\%) & \\
\hline 0.0 & 0.0000 & 0.0000 & 0.0000 & 0.0000 & 0.0000 & 0.0000 & 0.0000 \\
\hline 0.5 & 3.1181 & 0.0289 & 3.1173 & 0.0032 & 3.1172 & 0.0000 & 3.1172 \\
\hline 1.0 & 6.1595 & 0.0292 & 6.1578 & 0.0016 & 6.1577 & 0.0000 & 6.1577 \\
\hline 1.5 & 9.0491 & 0.0276 & 9.0467 & 0.0011 & 9.0466 & 0.0000 & 9.0466 \\
\hline 2.0 & 11.7160 & 0.0282 & 11.7128 & 0.0009 & 11.7127 & 0.0000 & 11.7127 \\
\hline 2.5 & 14.0945 & 0.0291 & 14.0905 & 0.0007 & 14.0904 & 0.0000 & 14.0904 \\
\hline 3.0 & 16.1259 & 0.0292 & 16.1213 & 0.0006 & 16.1212 & 0.0000 & 16.1212 \\
\hline 3.5 & 17.7602 & 0.0299 & 17.7551 & 0.0011 & 17.7550 & 0.0006 & 17.7549 \\
\hline 4.0 & 18.9574 & 0.0306 & 18.9517 & 0.0005 & 18.9516 & 0.0000 & 18.9516 \\
\hline 4.5 & 19.6875 & 0.0305 & 19.6817 & 0.0010 & 19.6815 & 0.0000 & 19.6815 \\
\hline 5.0 & 19.9306 & 0.0191 & 19.9270 & 0.0010 & 19.9268 & 0.0000 & 19.9268 \\
\hline
\end{tabular}


Table 3. The variation of temperature at $x=0.0$

\begin{tabular}{cccc}
\hline $\mathrm{Y}$ & Present solution & Analytical solution & Error (\%) \\
\hline 1.0 & 0.5000215 & 0.5000000 & $4.30 \times 10^{-3}$ \\
2.0 & 0.2951657 & 0.2951672 & $-5.08 \times 10^{-4}$ \\
4.0 & 0.1559584 & 0.1559583 & $6.41 \times 10^{-5}$ \\
8.0 & 0.0791669 & 0.0791668 & $1.26 \times 10^{-4}$ \\
16.0 & 0.0397371 & 0.0397370 & $2.52 \times 10^{-4}$ \\
32.0 & 0.0198879 & 0.0198879 & 0.00 \\
64.0 & 0.0099464 & 0.0099464 & 0.00 \\
128.0 & 0.0049735 & 0.0049735 & 0.00 \\
256.0 & 0.0024868 & 0.0024868 & 0.00 \\
\hline
\end{tabular}

